\title{
PARK2 Microduplication: Clinical and Molecular Characterization of a Further Case and Review of the Literature
}

\author{
Orazio Palumbo ${ }^{a}$ Pietro Palumbo ${ }^{a}$ Maria P. Leone ${ }^{a, b}$ Raffaella Stallone ${ }^{a}$ \\ Teresa Palladino $^{a}$ Marcella Vendemiale $^{c}$ Stefano Palladino $^{d}$ \\ Francesco Papadia ${ }^{e}$ Massimo Carella ${ }^{a}$ Rira Fischetto ${ }^{e}$ \\ a Laboratorio di Genetica Medica, IRCCS Casa Sollievo della Sofferenza, San Giovanni Rotondo, bipartimento di \\ Scienze del suolo, della pianta e degli alimenti, Università degli Studi di Bari 'Aldo Moro', 'Servizio di Psicologia

 \\ Metaboliche Genetica Medica, PO Giovanni XXIII, A.O.U. Policlinico Consorziale, Bari, Italy
}

\section{Key Words}

Neurodevelopmental disorders · PARK2 - SNP array analysis

\begin{abstract}
We report on a patient with psychomotor deficits, language delay, dyspraxia, skeletal anomalies, and facial dysmorphisms (hirsutism, right palpebral ptosis, a bulbous nasal tip with enlarged and anteverted nares, and a mild prominent antihelix stem). Using high-resolution SNP array analysis, we identified a $0.49-\mathrm{Mb}$ microduplication in chromosome $6 \mathrm{q} 26$ inherited from the mother involving the PARK2 gene: arr[hg19] $6 q 26(162,672,821-163,163,143) \times 3$ mat. To the best of our knowledge, this is the third patient to date described in whom a 6 q26 microduplication encompassing only the PARK2 gene has been reported in medical literature. The PARK2 gene is a neurodevelopmental gene that was initially discovered as one of the causes of autosomal recessive juvenile Parkinson disease and subsequently reported to be linked to autism spectrum disorders and attention-deficit hyperactivity disorders. We provide an overview of the literature on PARK2 microduplications and further delineate the associated pheno-
\end{abstract}

type. Taken together, our findings confirm the involvement of this gene in neurodevelopmental disorders and are useful to strengthen the hypothesis that, although with variable expressivity and incomplete penetrance, the PARK 2 microduplication is associated with a new emerging neurodevelopmental delay syndrome. However, clinical and molecular evaluations of more patients with the microduplication are needed for full delineation of this syndrome.

(c) 2016 S. Karger AG, Basel

PARK2, located in 6q26, encodes parkin, a cytoskeletal-associated protein expressed in neuronal processes and cell bodies of the midbrain, basal ganglia, cerebral cortex, and cerebellum [Huynh et al., 2000]. Parkin is an E3 ubiquitin-protein ligase and transcriptional repressor of p53 [Kitada et al., 1998; da Costa et al., 2009].

Point mutations in PARK2 cause autosomal recessive juvenile Parkinson disease (MIM 600116) [Kitada et al., 1998], while microdeletions of the same gene have been described in patients with autism spectrum disorders. In 2009, Glessner et al. identified 7 unrelated carriers of a

\section{KARGER}

E-Mail karger@karger.com

www.karger.com/msy
(C) 2016 S. Karger AG, Basel

$1661-8769 / 16 / 0075-0282 \$ 39.50 / 0$
Orazio Palumbo, $\mathrm{PhD}$

Laboratorio di Genetica Medica IRCCS Casa Sollievo della Sofferenza IT-71013 San Giovanni Rotondo (Italy)

E-Mail o.palumbo@operapadrepio.it 
chromosome 6q26 microdeletion affecting the PARK2 gene in a cohort of 859 patients with autism spectrum disorders by using a high-resolution microarray technology (Affymetrix 500K SNP-array, Santa Clara, Calif., USA). The same copy number variation (CNV) was not observed in 1,409 healthy controls. In contrast, little is known about the reciprocal microduplication; only 2 patients are reported in literature thus far. Scheuerle and Wilson [2011] reported the first interstitial microduplication of $6 \mathrm{q} 26$ (161 kb in size) encompassing the PARK2 gene, detected by chromosome microarray analysis and confirmed by FISH, in a boy with a diagnosis of Asperger syndrome and cognitive impairment. In addition, the patient showed facial dysmorphisms, relative macrocephaly and was hypotonic. By performing FISH, the authors were able to identify the same rearrangement in the mother and hypothesized the duplication of PARK2 as responsible for an emerging microduplication syndrome. Some years later, Mariani et al. [2013] described a similar clinical and molecular situation corroborating the hypothesis that microduplication of the PARK2 gene could play a role in the etiology of neurodevelopmental anomalies in humans. They reported an 8-month-old female with a 314-kb copy number gain involving the PARK2 gene. She presented with developmental delay, eye movement abnormalities, and axial hypotonia with peripheral hypertonia. The same microduplication has been found in her mother, highlighting the possible incomplete penetrance and/or variability of clinical expression of the PARK2 microduplication. In this paper, we report on the third case bearing a microduplication in chromosome 6q26 encompassing PARK2. The same rearrangement has been identified in the healthy mother. The patient is a 5-year and 10-month-old boy with neurodevelopmental disorders (NDDs), facial dysmorphisms, and skeletal anomalies.

\section{Patient and Methods}

\section{Clinical Report}

The index patient is the first and only child of unrelated healthy Caucasian parents. The boy was referred to our clinic at 5 years and 10 months of age for evaluation of neurodevelopmental delay (psychomotor and language delays), dyspraxia, and unusual facial features of unknown cause. He was born after a full-term uncomplicated pregnancy. The maternal and paternal ages were 38 and 36 years, respectively, at the time of his birth. Family history was unremarkable for neurological disorders or developmental delay. In addition, there was no history of exposures to teratogenic agents, illness, or drugs. Birth weight was 3,500 g (50th-75th percentile) and birth length was $51 \mathrm{~cm}$ (50th-75th percentile); his head circumference was not documented. There were no notable prenatal or immediate postnatal problems; no feeding difficulty was noted at any time.

The development milestones were somewhat slow, with a major delay in speech acquisition: he started walking at age 16 months, but had not yet started to speak at 18 months. At 3 years of age, psychological assessment (Griffiths Mental Developmental Scales; Wechsler Preschool and Primary Scale of Intelligence-III/WPPSIIII; Development Test of Visual-Motor Integration/V.M.I) was performed, resulting in quite normal psychomotor development, severe impairment in expressive language function, and visuomotor coordination difficulties.

Anthropometric measurements at the age of 5 years and 10 months were as follows: weight $21 \mathrm{~kg}$ (50th-75th percentile), and height $110 \mathrm{~cm}$ (10th-25th percentile). Physical examination showed hirsutism, right palpebral ptosis, a bulbous nasal tip with enlarged and anteverted nares, mild prominent antihelix stem, and slightly reduced muscle tone (fig. 1A). The patient had skeletal anomalies including kyphosis, flat feet and a skeletal age of 4 years. Extensive laboratory investigation followed, including a hearing test, cardiac echocardiogram and electroencephalogram, blood routine test and metabolic screen, all proved normal. MRI of the brain showed mild dilation of the occipital horns in lateral ventricles (fig. 1B), while radiographs revealed dorsal and lumbar spine scoliosis (fig. 1C). Conventional karyotyping yielded normal results $(46, \mathrm{XY})$.

At 6 years of age, a novel neuropsychomotor evaluation (WPPSI-III; Child Behaviour Checklist/CBCL; Projective Tests paper and pencil) demonstrated the impairment predominantly in his verbal domain, expressive language and fine motor skills, compared with his gross motor skills and sociality. Moreover, deficiency in visual-spatial discrimination and motor coordination were evident. Borderline anxiety and depression also emerged.

SNP Array Analysis

DNA samples of the patient and his parents were isolated from peripheral blood leukocytes by using BioRobot EZ1 (Quiagen, Solna, Sweden). DNA concentration and purity were determined with a ND-1000 Spectrophotometer (NanoDrop Technologies, Berlin, Germany), and high-resolution SNP array analysis was performed using CytoScan ${ }^{\mathrm{TM}} \mathrm{HD}$ Array (Affymetrix) as previously described [Palumbo et al., 2014]. All nucleotide positions were based on the February 2009 human reference sequence, assembly GRCh37/ hg19, produced by the Genome Reference Consortium.

\section{Results}

SNP array analyses of the proband and his parents showed a copy number gain, in the patient and his mother, in chromosome band $6 \mathrm{q} 26$ of $\sim 0.49 \mathrm{Mb}$ containing the PARK2 gene (fig. 2). According to the International System for Human Cytogenetic Nomenclature [ISCN, 2013], the molecular karyotype of the patient (showing both the minimum and maximum extent of the microduplication) was defined as follows: $\operatorname{arr}[\mathrm{hg} 19] \mathrm{6q} 26(162,664,364 \times 2$, $162,672,821-163,163,143 \times 3,163,163,594 \times 2$ )mat (fig. 2). No other pathological CNVs have been identified, excluding well-known benign CNVs (data not shown). 
Fig. 1. A Facial appearance of our patient at the time of clinical evaluation (5 years and 10 months). Notable findings include hirsutism, right palpebral ptosis, a bulbous nasal tip with enlarged and anteverted nares, and a mild prominent antihelix stem. Brain MRI (B) and radiograph (C) show mild dilation (indicated by arrows) of the occipital horns in lateral ventricles, dorsal and lumbar spine scoliosis, respectively.


Fig. 2. Results of the SNP array analysis ( $\log _{2}$ ratio) showing the $0.49-\mathrm{Mb}$ interstitial microduplication in our patient, inherited from the mother, in the region $6 \mathrm{q} 26$ (chr6: 162,672,821-163,163,143; hg19).



\section{Discussion}

The widespread use of chromosome microarray analysis in the diagnostic work-up of patients with neurodevelopmental disorders has led to the discovery of new microdeletion/microduplication syndromes and the identification of the candidate genes responsible for the clinical phenotypes [Cooper et al., 2011]. Interstitial microduplication in chromosome $6 \mathrm{q} 26$ encompassing the PARK2 gene are very rare; to the best of our knowledge, only 2 patients are reported in the literature [Scheuerle and Wilson, 2011; Mariani et al., 2013]. Both patients were diagnosed by chromosome microarray analysis and displayed syndromic NDDs. The authors suggested that the duplication of the PARK2 gene could be a new emerging microduplication syndrome.

Here, we report a further patient carrying a maternal interstitial $0.49-\mathrm{Mb}$ microduplication in chromosome 6q26 containing the PARK2 gene. The phenotype of our patient and 2 previously described cases with PARK 2 microduplication is presented in table 1 .

All patients showed a common syndromic neurodevelopmental phenotype characterized by developmental delay (3/3), psychomotor delays/intellectual disability (3/3), hypotonia (3/3), and language impairment (2/3). In addition, the patient reported by Scheuerle and Wilson [2011] had a diagnosis of Asperger syndrome, whereas our patient showed dyspraxia. These predominant neurodevelopmental manifestations provide further clinical implications of the role of PARK2 microduplication in the etiology of NDDs. Since the patient described by Mariani et al. [2013] was only 8 months old, we cannot determine if she will develop autistic features, language and cognitive deficits, and/or dyspraxia. Therefore, some clinical features associated with the PARK2 duplication, could simply be underestimated to date, as they can be absent in patients which are too young to show such symptoms. An alternative hypothesis is that the absence of autism in our patient 
Table 1. Clinical and molecular findings in the present case and other reported patients with overlapping $6 \mathrm{q} 26$ microduplications

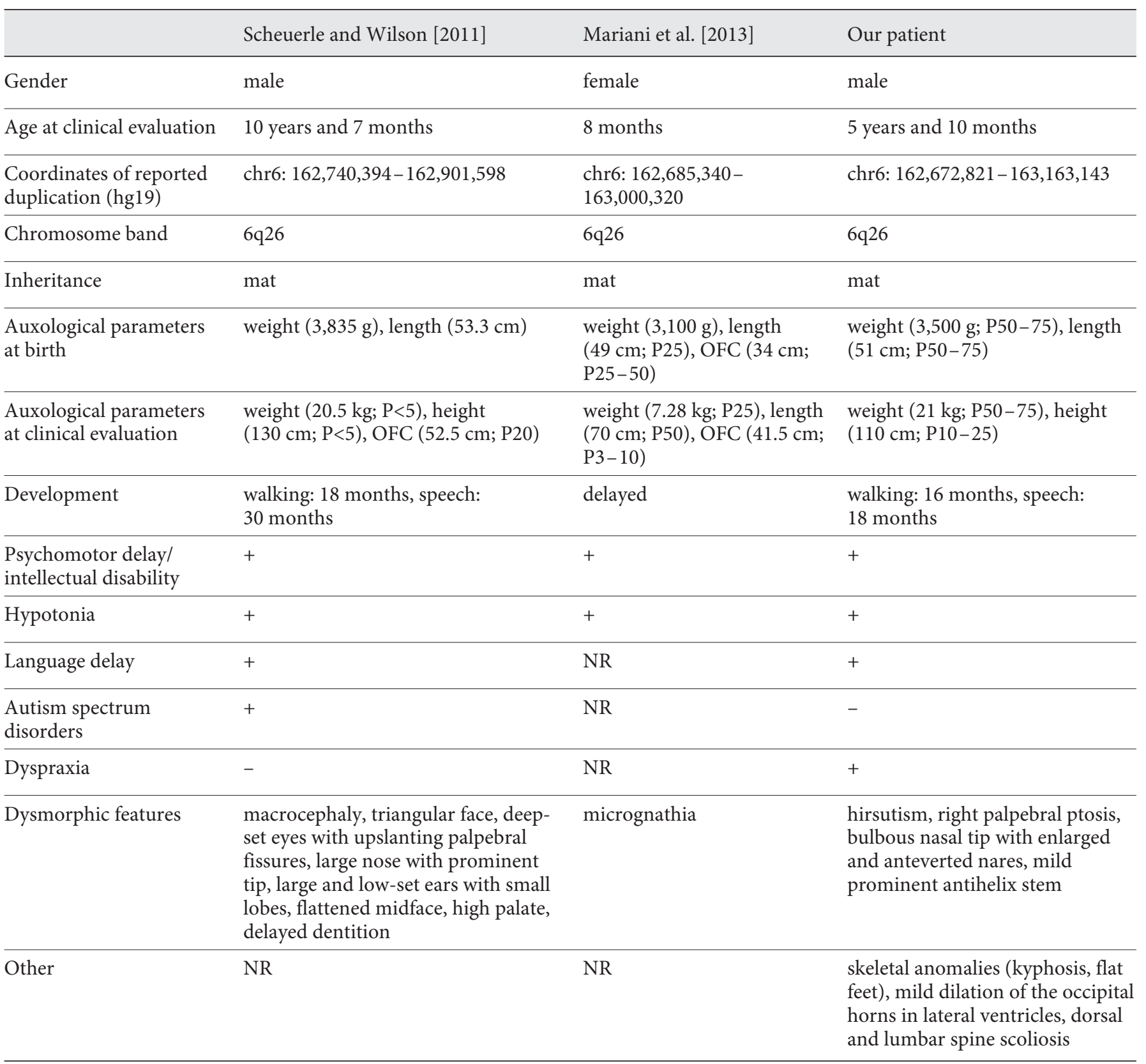

mat $=$ Inherited from the mother; $\mathrm{NR}=$ feature not reported $\mathrm{OFC}=$ occipital frontal circumference; $\mathrm{P}=$ percentile; $+=$ feature present; - = feature absent.

and dyspraxia in the patient described by Scheuerle and Wilson [2011] could be due to variable expressivity and/ or incomplete penetrance of $P A R K 2$ microduplication, as it has been suggested for other microduplication syndromes [Berg et al., 2010]. This hypothesis is corroborated by the fact that in all patients (our and both those previously reported) to date described in the literature, the 6q26 microduplication has been detected also in the healthy mothers. Considerung these clinical and molecular evidences, we suggest a regular neurological evaluation in patients carrying a 6q26 microduplication encompassing PARK2. Additional cases with known inheritance pattern of PARK2 microduplication will provide more evidence in genotype-phenotype correlation. 
Dysmorphic facial features are aspecific (macrocephaly, triangular face, deep-set eyes with upslanting palpebral fissures, large nose with prominent tip, large and low-set ears with small lobes, flattened midface, high palate, and delayed dentition) in the boy reported by Scheuerle and Wilson [2011] and absent (except for micrognathia) in the patient described by Mariani et al. [2013]. Also the dysmorphic features observed in our patient, including hirsutism, right palpebral ptosis, bulbous nasal tip with enlarged and anteverted nares, and mild prominent antihelix stem are not impressive, making this emerging microduplication syndrome not immediately recognizable and diagnosable, and we think that this is mainly due to the small number of patients reported in the literature. Therefore, investigating more patients carrying a PARK2 microduplication with a detailed clinical characterization may allow us to identify a possible consistent pattern of clinical recognizable features useful to suggest a diagnosis. Finally, in our patient, from a clinical point of view, skeletal anomalies in the form of kyphosis and flat feet, mild dilation of the occipital horns in lateral ventricles as well as dorsal and lumbar spine scoliosis have been observed, unreported in cases previously described and for this reason useful to expand the clinical spectrum associated with the 6q26 microduplication.

In conclusion, we report a third patient with a $6 \mathrm{q} 26$ microduplication inherited from the mother, encompassing only the PARK2 gene and an overlapping syndromic neurodevelopmental phenotype with the 2 previously described patients. This additional patient corroborates the existence of a new emerging microduplication syndrome characterized by developmental and psychomotor delays/intellectual disability, hypotonia, and language impairment. Further cases with microduplication encompassing only the PARK2 gene will contribute to the full clinical characterization of the new microduplication syndrome of $6 q 26$. Finally, given the variable expressivity of some of its clinical features and the clear incomplete penetrance of this new syndrome, we recommend a careful neurological assessment of patients with PARK2 microduplication and, if the duplication is inherited, a detailed clinical evaluation of the parent who transmitted the rearrangement as he/she may have a subtler phenotype than the proband. This is significant given the associated high risk of recurrence.

\section{Acknowledgment}

We are grateful to the patient and his family for agreeing to take part in this study. This study was supported by grants from the Italian Ministry of Health (Ricerca Corrente 2016 and RF201102350693) to M.C.

\section{Statement of Ethics}

Written informed consent was obtained from the patient for publication of this paper and any accompanying images.

\section{Disclosure Statement}

The authors declare that they have no competing interests.

\section{References}

Berg JS, Potocki L, Bacino CA: Common recurrent microduplication syndromes: diagnosis and management in clinical practice. Am J Med Genet A 152A:1066-1678 (2010).

-Cooper GM, Coe BP, Girirajan S, Rosenfeld JA, $\mathrm{Vu} \mathrm{TH}$, et al: A copy number variation morbidity map of developmental delay. Nat Genet 43:838-846 (2011).

da Costa CA, Sunyach C, Giaime E, West A, Corti $\mathrm{O}$, et al: Transcriptional repression of p53 by parkin and impairment by mutations associated with autosomal recessive juvenile Parkinson's disease. Nature Cell Biol 11:13701375 (2009).

-Glessner JT, Wang K, Cai G, Korvatska O, Kim $\mathrm{CE}$, et al: Autism genome-wide copy number variation reveals ubiquitin and neuronal genes. Nature 459:569-573 (2009).
Huynh DP, Scoles DR, Ho TH, Del Bigio MR, Pulst S-M: Parkin is associated with actin filaments in neuronal and nonneural cells. Ann Neurol 48:737-744 (2000).

ISCN (2013): An International System for $\mathrm{Hu}-$ man Cytogenetic Nomenclature, Shaffer LG, McGowen-Jordan J, Schmid M (eds) (Karger, Basel 2013).

Kitada T, Asakawa S, Hattori N, Matsumine H, Yamamura Y, et al: Mutations in the parkin gene cause autosomal recessive juvenile parkinsonism. Nature 392:605-608 (1998).
Mariani M, Crosti F, Redaelli S, Fossati C, Piras R, et al: Partial duplication of the PARK2 gene in a child with developmental delay and her normal mother: a second report. Am J Med Genet B Neuropsychiatr Genet 162B:485-486 (2013).

Palumbo O, Fichera M, Palumbo P, Rizzo R, Mazzolla E, et al: TBR1 is the candidate gene for intellectual disability in patients with a $2 \mathrm{q} 24.2$ interstitial deletion. Am J Med Genet Part A 164A:828-833 (2014).

Scheuerle A, Wilson K: PARK2 copy number aberrations in two children presenting with autism spectrum disorder: further support of an association and possible evidence for a new microdeletion/microduplication syndrome. Am J Med Genet B Neuropsychiatr Genet 156B:413-420 (2011). 